\title{
LAS TEORIAS DEL MOVIMIENTO DE LOS PROYECTILES $Y$ DE LAS PARALELAS DE ARISTÓTELES A EINSTEIN
}

Albert Dou

\section{Introducción}

Según A. Koyre, "El logro más brillante conseguido por Descartes-fisico es sin duda, haber proporcionado una fórmula "clara y distinta» del principio de inercia; y haberla puesto en su lugar" (1966, p. 149). Es un logro repleto de fallos y aciertos, complicado en exceso y que puede verse en Koyré.' No es mi intención ocuparme aqui de la historia de este brillante resultado de Descartes; también omitiré la contribución de Gassendi, quien fue el primero en confirmar experimentalmente la ley de inercia y publicarla, como puede verse también en Koyré (I.c.). En un escrito de 1987 me ocupé de la extraordinaria importancia que tiene la ley de inercia en la historia de la teoría del movimiento de los proyectiles desde Aristóteles hasta Einstein; en cll relacioné la evolución de esta teoria con la evolución de la teoria de las paralelas desde Euclides hasta Einstein. Pues, me parecio, en efecto, que hay sorprendentes puntos de contacto entre ambas teorías. En estos dos últimos años he repensado el tema y me parece que puedo añadir algunos complementos que pueden ser interesantes.

En consecuencia, mi conferencia será una exposición paralela de las teorias del movimiento de los proyectiles y de las paralelas, centrada en tres cortos periodos. Asi pues, dividiré la exposición en tres secciones.

1) Limitándonos a lo que aqui interesa, exposición de la teoría del movimiento de los proyectiles de Aristóteles y muy brevemente de la teoria de las paralelas de Euclides. Ambas teorias nacen en el siglo 4 a. de $C$.

2) Cambio total de paradigma kuhniano y descubrimiento del concepto de curvatura, gracias a Galileo, Newton y Huygens (siglos 17 y 18); descubrimiento de las geometrias no euclidianas y concepto de curvatura de una superficie, gracias a Gauss, Bolyai, Lobachevski y Beltrami (siglos 18 y 19).

3) Formas simplicisimas que toman ambas teorias en la teoria de la relatividad general de Einstein (siglo 20).

Es especialmente notable el papel que desempeña el concepto general de curvatura en ambas teorias; y la recuperación por Einstein del movimiento natural introducido por Aristóteles, después de su preterición en la mecánica moderna creada por Newton.

\section{Siglo 4 a. de C.}

Aristóteles en el estudio de las disciplinas teóricas establece el concepto de ciencia. "Puede decirse siempre que [en una ciencia] naturalmente se dan estas tres cosas: aquello [gemus o materia sobre la que versa la ciencia] de lo que se demuestra algo, lo que se demuestra, y

\footnotetext{
1 ^. Koyré, Essmdios galileanos, Siglo veintiuno editores, primera edición espantola 1980.
} 
aquello [reglas de inferencia] mediante lo cual se demuestra".2 Esta noción de ciencia tuvo vigencia hasta Galileo, $y$ la tiene todavia por lo que se refiere a la necesidad de reglas de indiferencia y de unos primeros axiomas o postulados. Para Aristóteles la física es la ciencia que estudia las cosas que tienen una existencia separada y que están sujetas a cambio; o sea los cuerpos naturales, los cuales poseen en sí mismos un principio de reposo y movimiento. En cambio, las matemáticas estudian cosas que no están sujetas a cambio, pero que no tienen existencia separada.

\subsection{El movimiento de los proyectiles}

En la fisica, entre los movimientos (o cambios) de los cuerpos, Aristóteles considera especialmente el movimiento local, que define como cambio de lugar. Por tanto el movimiento local está sujeto al principio básico e incontrovertible de todo cambio, a saber que todo cambio tiene una causa eficiente.

Aristóteles divide el movimiento local de diversas maneras; asi, según la forma de la trayectoria del móvil, puede ser rectilineo, curvilineo y mixto; especialmente nos interesa aqui la división, según su adecuación al móvil, en natural, y no natural o violento. La definición de Aristóteles del movimiento natural depende estrechamente de varios principios de su fisica y cosmologia. Entre éstos, la existencia en el mundo sublunar de exactamente cuatro elementos fundamentales (tierra, agua, aire, fuego) caracterizados por dos pares de cualidades opuestas (frio-caliente, seco-húmedo). Existe una relación esencial de estos cuatro elementos con otro par de cualidades apuestas, a saber su gravedad-ligereza. Esta úttima cualidad y la existencia de un centro del mundo sublunar ocupado por la Tierra, estructuran el mundo sublunar en anillos esfëricos homogéneos concéntricos con centro común en la Tierra y ordenados según la gravedad-ligereza de los cuerpos que los ocupan; los más graves en el centro y los más ligeros (fuego) en la periferia, de modo que cada cuerpo tiene su anillo esfírico propio, hacia el que tiende si se encueatra apartado de êl.

He aqui el principio que rige el movimiento natural de un cuerpo no semoviente: un cuerpo que no esté influenciado por un agente y que no esté en su lugar propio se moverá con un movimiento, que llamaremos natural, hacia su lugar propio. Por ejemplo, la gota de agua que se forma en las nubes y que durante el movimiento de caida va actualizando su propia gravedad hasta actualizarla totalmente cuando llega al océano.

El movimiento violento obedece al siguiente principio: rodo movimiento violento requiere la acción de un agente, distinto del móvil mismo, que sea constantemente causa eficiente del mismo. Parece, pues, que el movimiento natural se debe a la naturaleza misma del móvil en cuanto éste se relaciona con el medio, aunque éste parece comportarse de manera totalmente pasiva.

Surge una seria dificultad al aplicar este principio al movimiento violento de los proyectiles. ¿Cuál es el agente o la causa eficiente o el motor que mueve la piedra arrojada cuando ya ha salido de la mano?

Sólo caben tres alternativas según Aristóteles: o bien el motor está a distancia del proyectil, o bien está en contacto con el proyectil y es exterior a él, o bien está en el interior del proyectil. Ahora bien, Aristóteles rechaza la acción a distancia y tampoco admite que el motor esté en el interior del proyectil; por tanto argumenta que el motor está en el exterior del proyec-

2 Véase Segundos analiticos 1, 10; 76a37 - 76b23. 
til y en contacto con él. A este efecto se le ha atribuido a Aristóteles una teoría de movimientos peristálticos que producirian el efecto motor deseado; pero esta atribución es incortecta, como ha mostrado Prevosti (1985, pp. 334-335). Con todo, el razonamiento de Aristóteles, así como la teoria del impetus, después de muchos y largos argumentos, fueron final y definitivamente rechazados por Galileo.

En el movimiento del proyectil hay que conjugar el movimiento debido al tiro o lanzamiento inicial con al movimiento natural del mismo proyectil debido a su cualidad de grave. Pero Aristóteles no conoció la ley del paralelogramo para la composición de movimientos, y parece que conjugó los dos movimientos no simultánea sino sucesivamente. Primero el debido a la acción inicial, el cual por resistencia del aire o del medio va perdiendo su carácter motriz, y sólo cuando se ha extinguido su influencia, entra en acción la gravedad del cuerpo arrojado.

Notemos que manteniendo el principio general básico de todo movimiento, pueden darse otras dos alternativas mucho más radicales. La primera, que el movimiento rectilineo uniforme no es un cambio propiamente tal, sino meramente un estado de la materia, por ejemplo porque no consume energía, aunque "haya cambio de lugar". La segunda, que todo movimiento rectilineo uniforme sea considerado como movimiento natural. La primera alternativa corresponde al descubrimiento de la ley de inercia (Galileo); la segunda coincide con la explicación que se da en la teoría de la relatividad general (Einstein).

\section{I.2. El quinto postulado de Euclides.}

El libro de los Elementos, escrito por Euclides al final del siglo 4 a. de C., contiene la primera teoria de rectas paralelas. El primer libro de los Elementos constituye la geometría elemental que todavia hoy se enseña en la ensenanza obligatoria. La geometria plana estudia puntos, rectas y círculos y sus propiedades; y se fundamenta en axiomas o postulados de incidencia, de orden, de congruencia, un postulado de continuidad y finalmente un postulado que fundamenta la teoria de las rectas paralelas, y se llama postulado de Euclides.

Definición de rectas paralelas: Se dice que dos rectas situadas en un mismo plano son paralelas, sólo y cuando por más que se las prolongue en ambas direcciones, nunca se cortan (ni divergen a la vez en ambas direcciones).

Supongamos que tenemos un plano cualquiera y en él una recta arbitraria $r$ y un punto $P$ fuera de la recta $r$ pero por lo demás arbitrario. Sea $Q$ el pie de la perpendicular bajada desde $P$ sobre $r$, de modo que $Q$ es un punto de $r$ y la recta $P Q$ es perpendicular a $r$. Entonces, el postulado de Euclides es equivalente a la proposición siguiente: Por el pumto $P$ no pasa ninguna recta paralela a la recta r o a lo sumo pasa una sola.

Euclides en la proposición 1.17 y suponiendo que la línea recta es de longitud potencialmente infinita, demuestra que en todo triängulo la suma de dos ángulos cualesquiern es siempre menor que dos rectos. Con esta proposición I.17 demuestra en las proposiciones 1.27-1.29 que la recta trazada por $P$ perpendicularmente a la recta $P Q$ es en efecto la única paralela pasando por $P$ a la recta $r$.

\section{Siglos xvi1-xix}

\subsection{La ley de inercia}

La mecánica aristotélica y la misma noción de ciencia da un cambio cualitativo muy importante con las aportaciones de Galileo (1564-1642). En el centro de este cambio, o en términos actuales en el centro de la revolución cientifica por antonomasia, como pieza central del nuevo paradigma kuhniano está la ley de inercia. Galileo es quien la descubre y formula por primera vez. 
y la da ya a conocer en el Diálogo (1632) y luego en los Discorsi (1638), aunque con ligeras imperfecciones.

La nueva física moderna cristaliza en los Principia (1687) de Newton, y de ellos tomamos la formulación de la ley de inercia o primera ley del movimiento según Newton:

Todo cuerpo persevera en su estado de reposo o de movimiento uniforme y rectilineo, a menos que sea obligado por fuerzas impresas a cambiar de estado.

Unas pocas páginas más adelante, en el Corolario 5 demuestra: "Los movimientos relativos de los cuerpos incluidos en un cierto espacio son los mismos, tanto si este espacio está en reposo como si se mueve rígidamente con un movimiento uniforme y rectilineo". Equivale a decir que las leyes de la física moderna son invariantes respecto de cualquier sistema galileano, es decir cualquier sistema con movimiento uniforme y rectilíneo respeto de los ejes de Copérnico (origen en el centro de gravedad del sistema solar, plano de la ecliptica y un eje pasando por el punto vernal). Es el principio de relatividad de la fisica moderna (llamada también por algunos física clásica).

Galileo formula de forma contundente esta ley con un relato muy pintoresco, tomando como "cierto espacio" la "mayor estancia bajo cubierta de un gran navio" (Diálogo, $2^{2}$ jornada). El descubrimiento de la ley de inercia no fue fácil. Galileo confió en las matemáticas acercándose a Platón y separándose de Aristóteles, como parece desprenderse del Diálogo (Kóoyré, l.c. pp. 135-148, 264-278). Notemos dos imperfecciones en la teoria galileana de la ley de inercia: que la enuncia sóla para determinadas superficies planas, la del mar y la del plano inclinado; que en el mar el movimiento no seria exactamente rectilíneo, sino que describiría un círculo máximo. Gracias a la ley de inercia y a la ley del paralelogramo, también descubierta por Galileo, para la composición simultánea de movimientos, Galileo consigue deducir la trayectoria parabólica del proyectil lanzado por el plano inclinado; además, brillantemente y por primera vez en la historia de la ciencia, confirma esta deducción experimentalmente.

Newton, gracias al cálculo diferencial que él mismo construye, a la ley de inercia y a las otras dos leyes que introduce, crea, fundamenta y desarrolla la mecánica moderna. La segunda ley, $f=m \bar{a}$, o sea, llamando $\bar{s}$ al espacio recorrido por el punto material móvil de masa $m$, $\mathrm{f}=m \cdot\left(d \xi / \mathrm{dt}^{2}\right)$, y teniendo en cuenta que la curvatura depende linealmente de las derivadas segundas, se pone de manifiesto la importancia de la curvatura en el estudio de las trayectorias de los puntos materiales móviles. Asi, un punto material móvil, aunque su movimiento sea uniforme en cuanto que su velocidad absoluta sea constante, necesita de fuerzas impresas si su movimiento no es rectilíneo. Únicamente cuando la curvatura es idénticamente nula no hay fuerzas impresas y así, cuando ă=0, recuperamos el movimiento inercial. Notemos finalmente que el movimiento inercial escapa de las tres posibilidades aristotélicas y constituye una alternativa más radical.

\subsection{Las geometrias no euclidianas (siglos XVIII-XIX)}

Hasta la segunda mitad del siglo XvIII no hubo ningún geómetra que dudara de la veracidad del quinto postulado de Euclides; y no hubo geómetra importante que no diera una pseudodemostración del mismo. Además, el postulado, que en primer lugar era una proposición geométrica, implicaba también, según la filosofia aristotélica y la escolística, una ley física. El primero en cambiar sustancialmente esta situación fue el lógico moderno y geómetra G. Saccheri con su Euclides ab onni naevo vindicatus (Euclides reivindicado de toda mancha, 1733). Sustituye 
adecuadamente el quinto postulado por las tres únicas hipótesis posibles que le pueden sustituir y asi crea las tres geometrias del ángulo obtuso, del ángulo recto (que coincide con la de los Elementos de Euclides) y la del ángulo agudo, demostrando correctamente numerosos y profundos teoremas; comete un paralogismo de ignorantia elenchi y cree que ha demostrado el quinto postulado, pero en realidad fue la última pseudodemostración.

Saccheri introdujo el método que a la larga inexorablemente conduciria al descubrimiento de la existencia de la primera geometría no euclidiana, a saber, la que Saccheri llamó del ángulo agudo. El primero en descubrirla es Gauss y unos pocos lustros más tarde la descubren y publican J. Bolyai (1832) y N. Lobachevski (1829). Con ello queda demostrado que el quinto postulado de Euclides no es evidente, sino que existe por lo menos otra alternativa, a saber la existencia de una geometría del ángulo agudo, que más tarde (1871) Klein llamará geometria hiperbólica. Más tarde Riemann demostrará la existencia de una tercera geometria, la que Saccheri llamó del ángulo obtuso y que Klein (1871) llamará eliptica, aunque para ello ha de suponer que la recta es cerrada y de longitud finita.

Gauss crea (1827) el formidable concepto de curvatura en un punto cualquiera de una superficie, que abre paso a las geometrias rimanianas y a la relatividad general. Mediante este nuevo concepto de curvatura gaussiana Beltrami demostrará (1868) que la geometria intrinseca de puntos y geodésicas de una superficie de curvatura gaussiana constante es idéntica a las geometrias elíptica, euclidiana o hiperbólica según que la curvatura sea mayor, igual o menor que cero.

Merece notarse el importante y decisivo papel que desemperia la curvatura tanto en las trayectorias de puntos materiales móviles como en la teoria de las paralelas. En realidad este hecho refleja la progresiva aplicación del cálculo diferencial y el que la curvatura dependa linealmente de las derivadas segundas de las funciones que definen las trayectorias o las superficies respecto de las variables espaciales independientes. Concurre además que las derivadas segundas formalizan el crecimiento del crecimiento.

\section{Las teorías de las paralelas y del movimiento}

\section{de los proyectiles en la relatividad general (siglo $\mathrm{xx}$ )}

Sólo habian transcurrido dos siglos y medio cuando el experimento de Michelson-Morley (1887), con el que se demuestra la invariancia de la velocidad de la luz en el vacío, cuestiona seriamente la ley galileana de inercia y obliga a considerar el espacio-tiempo como un todo de cuatro dimensiones que no admite una división racional objetiva si quieren salvaguardarse la constancia de la velocidad de la luz y el nuevo principio inercial de la relatividad especial. Así, por obra de Einstein nace la teoria de la relatividad especial y las formulas de Lorentz para pasar de un sistema inercial a otro. En particular desaparece el concepto de simultaneidad absoluta.

Con todo, la solución al principio de relatividad dada por la teoria de la Relatividad especial aparece como insatisfactoria principalmente por dos razones. Primero, el mismo Newton. Leibniz y especialmente Mach ponen objeciones a la mecánica de Newton; el último objeta en particular que en la mecánica newtoniana el espacio-tiempo absoluto se comporta como agente y actúa, pero que no liay posibilidad de que se actúe sobre él; lo cual vale también para el espacio-tiempo de la relatividad especial. Segundo, Einstein objeta que no aparece porque los sistemas inerciales de la relatividad especial han de resultar privilegiados respecto a cambios más generales de coordenadas curvilineas. Consecuentemente, Einstein, basándose en las teorias de la acción a través de un medio como en el campo electromagnético y en la posibilidad 
bien justificada experimentalmente de identificar la masa inerte con la masa gravitatoria, introduce el principio de equivalencia, a saber, que los resultados de experimentos locales en un sistema de coordenadas en caída libre son independientes del movimiento del sistema de referencia, siempre y cuando el movimiento sea de caida libre.

3.l. La teoria de la relatividad general no aporta nada nuevo e importante a la teoria de las paralelas después de Beltrami y Klein. Recordemos que esta teoria se reftere primariamente a un plano, o conjunto de puntos y rectas que satisfacen unos postulados de incidencia, orden y congruencia (que dependen ligeramente de cual de las tres geometrias elementales se defina), un postulado de continuidad y un postulado de paralelas. Este puede ser de no existencia de paralelas (imponiendo por ejemplo que dos rectas se corten siempre), los cuales definen la geometría eliptica; o bien el postulado de que la longitud de la recta sea potencialmente infinita, y estos postulados definen la geometria hiperbólica (con dos paralelas a una recta por un punto fuera de ella); si además al postulado de infinitud de la recta añadimos el quinto postulado de Euclides, entonces queda definida la geometría euclidiana.

Beltrami demostró que la teoría de las paralelas puede extenderse a superficies de curvatura gaussiana constante, pues identificando las geodésicas con las rectas de un plano resulta que la geometria de las rectas y puntos de la superficie de curvatura constante coincide isométricamente con las mencionadas geometrias, aunque quizís sea sólo en regiones conexas de la superficie plana. Asi, si la curvatura es positiva, se obtiene una geometria eliptica; si es negativa se obtiene una geometria hiperbólica; y si la curvatura es nula se obtiene una geometria euclidana. Por tanto, en la relatividad general el paralelismo se encuentra en superficies de curvatura gaussiana constante, sumergidas en una variedad tridimensional espacial correspondiente a un valor determinado del tiempo.

3.2. El proyectil, según la teoria de la relatividad general, recorre una trayectoria que considerada en sí misma coincide, según el principio de equivalencia, con la trayectoria de un cuerpo en caida libre según las leyes de la mecánica neutoninna. Además esta coincidencia, y de acuerdo con el principio de equivalencia, determina la métrica o sea el tensor fundamental de la correspondiente variedad cuatridimensional del espacio-tiempo. Ahora bien, se demuestra sin dificultad que esta trayectoria del proyectil, referida a esta variedad cuatridimensional dotada de la métrica asi determinada, es un geodésica del espacio-tiempo.

Por consiguiente, recordando el concepto aristotélico de movimiento natural, parece que estas trayectorias de los proyectiles en su correspondiente espacio-tiempo de la relatividad general deberian ser especificados como movimientos naturales; pues, fuera del impulso inicial no necesitan motor; y por otra parte se deben exclusivamente a la relación del proyectil con el medio y su cosmología. Se recupera asi un interesante concepto aristotélico, que habia sido preterido por la dinámica moderna neutoniana. 